\title{
The Order of the Fullerene
}

\section{Roy ${ }^{1}$, M Olmstead $^{2}$, A Balch ${ }^{1}$ \\ ${ }^{1}$ University of California, Davis, Davis, CA, ${ }^{2}$ Dept of Chemistry, Univ of California Davis mriroy@ucdavis.edu}

The high symmetry of empty cage as well as endohedral fullerenes leads to orientational disorder in crystal structures. Often the extent of disorder prevents visibility of clean bonds and accurate measurement of bond lengths. This issue is especially cumbersome when a higher fullerene has a number of possible cage isomers, each of which is identifiable only through high-resolution single crystal data. A solution to the problem can be found in the phenomenon of cocrystallization - harnessing the non-covalent interaction between the fullerene and a second molecule (the cocrystallization agent) for ordering the unwieldy fullerene. In this systematic study, we crystallographically characterize the ordering interaction between C60 (the model fullerene) and late transition metal octaethylporphyrin [MII(OEP)] units (the chosen cocrystallization agents). Minor variations in the cocrystallization setup can dramatically influence the conformation of porphyrin molecules as well as the stoichiometry of their combination with $\mathrm{C} 60$. Such factors are examined, and their effects described in this work.

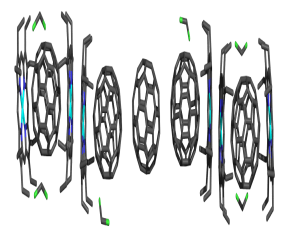

Figure 1

Acta Cryst. (2020). A76, a56 